\title{
Corela
}

Cognition, représentation, langage

HS-21 | 2017

Linguistique de corpus : vues sur la constitution, l'analyse et l'outillage

\section{Du rapport micro-diachronique futur simple/futur périphrastique en français moderne. Étude des variables temporelles et aspectuelles}

Lotfi Abouda et Marie Skrovec

\section{OpenEdition}

\section{Journals}

Édition électronique

URL : http://journals.openedition.org/corela/4804

DOI : $10.4000 /$ corela.4804

ISSN : 1638-573X

Éditeur

Cercle linguistique du Centre et de l'Ouest - CerLICO

Référence électronique

Lotfi Abouda et Marie Skrovec, « Du rapport micro-diachronique futur simple/futur périphrastique en français moderne.

Étude des variables temporelles et aspectuelles », Corela [En ligne], HS-21 | 2017, mis en ligne le 30 janvier 2017, consulté le 30 avril 2019. URL : http://journals.openedition.org/corela/4804 ; DOI 10.4000/corela.4804

Ce document a été généré automatiquement le 30 avril 2019

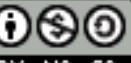

Corela - cognition, représentation, langage est mis à disposition selon les termes de la licence Creative Commons Attribution - Pas d'Utilisation Commerciale - Partage dans les Mêmes Conditions 4.0 International. 


\title{
Du rapport micro-diachronique futur simple/futur périphrastique en français moderne. Étude des variables temporelles et aspectuelles
}

\author{
Lotfi Abouda et Marie Skrovec
}

\section{Introduction}

1 Malgré un nombre conséquent de travaux, de nombreuses questions demeurent posées concernant la distribution en français moderne des deux formes du futur, i.e. le futur périphrastique (FP) et le futur simple (FS). Si certains auteurs (Mauger (1968), Togeby (1982), Fleischman (1983), Barcelo (2007), etc.) insistent sur le processus de grammaticalisation en cours qui conduirait à terme à la disparition du FS au profit de la forme périphrastique, de création romane plus récente, d'autres auteurs défendent l'hypothèse d'une complémentarité synchronique entre les deux formes, chacune se spécialisant, et donc se maintenant dans des configurations linguistiques spécifiques. A l'intérieur de chacune de ces tendances, on note des divergences importantes entre différents points de vue. Chez les premiers, se pose encore notamment la question de savoir si le FP connait un processus de grammaticalisation, formel et conceptuel, analogue à celui qu'a connu le FS ou s'il emprunte un chemin de grammaticalisation différent. Chez les seconds, la nature exacte de la complémentarité varie considérablement selon les auteurs, oscillant entre opposition aspecto-temporelle, comme chez Jeanjean (1988) et Bilger (2001), syntaxique, comme chez Emirkanian \& Sankoff (1986), modale comme chez Confais (1990), Maingueneau (1994) et Laurendeau 
(2000), voire diaphasique (i.e. de registre de langue), comme chez Arrivé, Gadet \& Galmiche (1986) ou encore Collier (2013).

Nous nous proposons de contribuer à ce débat, en nous appuyant sur un sous-corpus extrait des Enquêtes sociolinguistiques à Orléans (ESLO) ${ }^{1}$. Outre sa taille importante (actuellement autour de 7 millions de mots) qui permet la composition d'un sous-corpus équilibré, ESLO a en effet la particularité d'avoir été collecté en deux phases, à 40 ans d'intervalle, donnant ainsi naissance à deux sous-corpus comparables, mais diachroniquement différenciés, ESLO1 (1968-1971) et ESLO2 (2008-). Le regard microdiachronique que ces données documentées rendent possible nous semble particulièrement éclairant concernant le rapport FP/FS.

Dans une étude précédente consacrée aux changements survenus en micro-diachronie dans la distribution entre FS et FP en français moderne (Abouda \& Skrovec, 2015), nous avons mis en évidence une forte augmentation du FP corrélée à un affaissement du FS en l'espace de 40 ans $^{2}$, et montré que cette inversion du rapport de force s'observe tant pour les emplois proprement temporels de futurité que pour les emplois modaux (i.e. qui ne localisent pas le procès dans l'avenir et prennent en charge d'autres valeurs comme l'atténuatif ou le conjectural par exemple). Un examen plus précis des emplois modaux nous a ensuite fait découvrir une situation contrastée où coexistent différents cas de figure, qui permettent d'éclairer la question complexe de la concurrence ou de la complémentarité du FP face au FS. Nous nous proposons dans le présent travail d'étudier la distribution FS/FP des emplois temporels, i.e. qui localisent le procès dans un moment ultérieur au repère référentiel, en nous concentrant cette fois sur les variables temporelle et aspectuelle.

Après une première partie méthodologique, où il sera notamment question de présenter les données et la démarche suivie pour leur exploitation, il s'agira dans la deuxième partie de détailler le schéma d'annotation et de présenter les différentes étiquettes, dont les principales valeurs seront illustrées par des exemples du corpus. En troisième partie, après la présentation des principales tendances statistiques générales, nous tenterons d'identifier le type de rapport micro-diachronique entre le FP et le FS à l'œuvre dans les emplois chronologiques, en exploitant les propriétés sémantiques annotées.

\section{Corpus et méthodologie}

\subsection{Le sous-corpus}

5 Cette étude se base sur l'exploration d'un corpus échantillonné d'environ 1 million de mots (80 heures et 51 minutes), composé, à parts égales, d'extraits d'ESLO1 et d'ESLO2. Si cet équilibrage micro-diachronique, vu les données disponibles lors de la constitution du sous-corpus, n'a pas pu être accompagné d'un équilibrage diaphasique entre (et à l'intérieur de) chacun des deux extraits ESLO1/2, nous avons tout de même réussi, dans un corpus disponible majoritairement constitué d'entretiens, à intégrer, à hauteur de $20 \%$, deux genres interactionnels de "contrôle », supposés se situer de part et d'autre d'une échelle diaphasique, i.e. les repas (interactions informelles en famille ou entre amis autour d'un repas) et les conférences (prise de parole monologale devant un auditoire en milieu universitaire, présentant un degré de formalité assez élevé). 
6 Le tableau ci-dessous récapitule les caractéristiques quantitatives (durée en minutes et nombre de locuteurs) du corpus et sa composition diachronique et diaphasique :

\begin{tabular}{|l|l|l|l|l|}
\hline & Genre & ESLO1 & ESLO2 & Corpus global \\
\hline \multirow{4}{*}{ Durée (en min.) } & Conférences & 192 & 186 & 378 \\
\cline { 2 - 5 } & Repas & 196 & 201 & 397 \\
\cline { 2 - 5 } & Entretiens & 2042 & 2034 & 4076 \\
\cline { 2 - 5 } & Total & 2430 & 2421 & 4851 (80h et 51min) \\
\hline Nombre de locuteurs & Entretiens & 30 & 30 & 60 \\
\hline
\end{tabular}

Tableau 1 : Constitution du corpus ${ }^{3}$

7 A l'intérieur de cette composition, s'est parallèlement posée la question de la sélection des enregistrements notamment pour les entretiens. S'agissant d'un corpus de données situées, nous avons essayé de garantir au mieux un équilibrage diastratique (entre ESLO1 et ESLO2 et à l'intérieur de chacun d'entre eux), en termes de profil de locuteurs, choisis selon les variables de sexe $(\mathrm{H} / \mathrm{F})$, d'âge (3 tranches) et de catégorie socio-professionnelle $(5 \text { classes })^{4}$. Ces différents équilibrages ne visent pas à atteindre une quelconque représentativité, mais à réduire les variables entre les deux extraits ESLO1/ESLO2 en sélectionnant des enregistrements pouvant offrir la meilleure comparabilité qualitative possible.

\subsection{Démarche}

8 Pour exploiter le corpus constitué, nous avons utilisé le logiciel d'analyse textométrique, $\mathrm{TXM}^{5}$, couplé au logiciel Treetagger ${ }^{6}$ qui permet de lemmatiser un corpus transcrit et d'identifier les formes verbales fléchies. Les listes d'occurrences des deux formes du futur extraites grâce à des requêtes $\mathrm{CQL}^{7}$ ont été exportées dans des tableaux CSV, afin d'y être corrigées manuellement (l'objectif était d'écarter les erreurs d'annotation, mais aussi, en ce qui concerne le FP, d'écarter les occurrences d'aller au sens lexical plein de verbe de mouvement, car la requête ne permet pas d'exclure ces dernières) et faire l'objet d'une annotation affinée, où chacune des occurrences a été sous-spécifiée pour un certain nombre de traits. L'étape finale de cette démarche a consisté à réinjecter sous TXM les listes d'occurrences (corrigées et annotées) ainsi que les étiquettes d'annotation forgées dans l'objectif d'explorer les données en textométrie en croisant approches quantitative et qualitative. Les tendances quantitatives dégagées, si elles sont quelquefois significatives en elles-mêmes, nous ont souvent obligés, pour être correctement interprétées, à un retour à des analyses qualitatives où il a été question d'identifier les éventuelles variables co-textuelles pertinentes dans la distribution FP/FS en relation avec les métadonnées situationnelles et diastratiques ${ }^{8}$. 


\subsection{Annotation}

Dans le sous-corpus constitué, 3335 occurrences de futur (FP et FS) ont été identifiées après correction. Elles se répartissent de la manière suivante :

\begin{tabular}{|l|l|l|l|}
\hline & ESLO1 & ESLO2 & ESLO1+2 \\
\hline FS & 979 & 461 & 1440 \\
\hline FP & 688 & 1207 & 1895 \\
\hline Total & 1667 & 1668 & 3335 \\
\hline
\end{tabular}

Tableau 2 : Fréquence des occurrences du FS et du FP dans ESLO 1 et 2

L'annotation des occurrences, qui repose sur un accord entre deux annotateurs experts (les auteurs de ce travail) 9 , a été menée en deux étapes successives, la première visant à identifier le type d'emploi et la seconde à sous-spécifier l'occurrence selon un certain nombre de traits, qui varient en partie en fonction du type d'emploi.

11 Lors de la première couche d'annotation, spécifiant le type de valeur sémantique exprimée, chaque occurrence a reçu une des trois valeurs possibles correspondant à l'une des trois classes d'emplois distinguées: en plus des deux emplois habituellement reconnus $^{10}$, i.e. les emplois modaux (étiquetés « $\mathrm{m} »$ ), et les emplois temporels de futurité (étiquetés «f»), nous avons admis une troisième classe, mixte, d'emplois (étiquetés «fm »), où la futurité s'accompagne d'un certain nombre de traits modaux. Ainsi, dans l'exemple suivant, le futur simple est à la fois futural (situe le procès dans l'avenir) et déperformatif (susceptible d'être remplacé par un impératif, il neutralise un effet performatif injonctif $)^{11}$ :

ESLO1_REPAS_273_B_10

va dire ça à mon frère

ben tu lui diras ce soir qu'il faut qu'il mette un coton avec de l'éther

12 Cette analyse, s'opposant ainsi à la conception la plus classique et la plus commune selon laquelle un emploi de futur ne pourrait être considéré comme modal que si'il a perdu tout trait de futurité, est rendue incontournable par nos choix méthodologiques ${ }^{12}$, et s'appuie sur un certain nombre de travaux spécifiques au futur (voir par exemple Confais (1990) et Maingueneau (1994), pour qui le futur serait porteur d'une tension modale systématique) et plus généraux, qui rejettent la dichotomie exclusive temps-modalité (voir Gosselin (2005) et Abouda (2015)).

13 La deuxième étape d'annotation diffère selon le type d'emploi. Pour les emplois étiquetés « $\mathrm{m}$ », étudiés dans un travail antérieur ${ }^{13}$, l'annotation (qui a été menée sur la totalité des emplois de ce type, soit 176 occurrences de FS et 455 occurrences de FP) a visé à identifier le type de modalité exprimée par chaque occurrence.

Dans la présente étude, nous nous proposons de nous concentrer exclusivement sur l'exploration des emplois futuraux, étiquetés « $\mathrm{f} »^{14}$. Longue et particulièrement délicate, l'annotation des emplois temporels n'a pu être menée à son terme que pour environ $30 \%$ des occurrences concernées (595 occurrences sur 2010), sélectionnées d'une manière qui 
garantisse le meilleur équilibrage possible ${ }^{15}$ entre genres interactionnels (conférences, entretiens et repas) :

\begin{tabular}{|l|l|l|l|l|l|}
\hline \multicolumn{2}{|l|}{} & CONF & ENT & REP & Total \\
\hline \multirow{2}{*}{ FP } & ESLO1 & 41 & 58 & 59 & 158 \\
\cline { 2 - 6 } & ESLO2 & 56 & 60 & 57 & 173 \\
\hline \multicolumn{2}{|l}{ Total FP } & 331 \\
\hline \multirow{2}{*}{ FS } & ESLO1 & 49 & 49 & 51 & 149 \\
\cline { 2 - 6 } & ESLO2 & 16 & 50 & 49 & 115 \\
\hline \multicolumn{2}{|l|}{ Total FS } \\
\multicolumn{2}{|l}{ Total FS + FP } & & 595 \\
\hline
\end{tabular}

Tableau 3 : Emplois temporels annotés

\section{Annotation des emplois temporels (« $f »)$}

15 L'architecture d'ensemble de l'annotation des emplois futuraux étiquetés « $\mathrm{f}$ » peut être présentée sous la forme arborescente suivante (pour le FP, mais le FS ne s'en distingue que sur certains points de détail) :

$$
\text { IN LII }
$$


Figure 1 : Structure arborescente de l'annotation des emplois futuraux pour le FP illustrant les principales valeurs par des exemples du corpus.

\subsection{Substituabilité FP/FS}

L'une des questions importantes qui se posent à propos de la distribution FS/FP concerne le caractère exclusif ou non de l'une ou l'autre des deux formes dans un contexte donné. Cette première propriété annotée vise à déterminer si une forme donnée du futur peut ou non être remplacée par l'autre forme ${ }^{16}$. Trois cas semblent devoir être distingués.

(i) La substitution (FP-FS ou FS-FP) s'avère impossible ${ }^{17}$

- FP non remplaçable par un FS, comme dans l'exemple suivant où le FP à valeur prospective prend le sens d'être sur le point de:

(1)ESLO1_ENT_001_C_3

Loc1 : et alors qu'est-ce que vous faites exactement comme travail en ce moment?

Loc2 : je suis boucher

Loc1 : oui oui oui et euh en quoi est-ce que ça consiste exactement?

Loc2 : eh bien je vais vous dire euh comment vous voyez ça ? pour moi c'est un mon

cas est peut-être un petit peu spécial pas parce que je vais vous expliquer euh j'ai une boucherie bien sûr euh qui m'appartient en propre mais en plus de ça depuis une dizaine d'années euh on ne peut pas dire le hasard mais enfin les relations ont fait que j'ai un de mes amis qui a ouvert deux supermarchés à Orléans et alors il m'a donné la gestion si vous voulez de ses deux rayons de boucherie de supermarché

19 - Dans le contexte spécifique de l'exemple suivant, le FS n'est pas remplaçable par un FP. Le conférencier, qui s'adresse à de futurs enseignants à la fin des annés 60 , déplore que l'apprentissage des langues étrangères se fasse aussi tardivement. Le procès, présenté comme la condition pour améliorer la motivation des élèves, est envisagé comme n'étant pas d'actualité, compte tenu des politiques éducatives de l'époque, ce qui rend ce contexte incompatible avec un $\mathrm{FP}^{18}$ :

(2) ESLO1_CONF_503_B_23

Loc1: je suis bien de votre avis plus on commencera tôt ce dressage et moins on pourra toujours on pourra de dispenser à ce niveau-là de chercher des motivations qui n'existent finalement pas oui n'est-ce pas hein c'est-à-dire que quand on commencera à apprendre l'anglais partout à huit ans eh bien vous o- vous organisez la actuel de ce qu'on appelle la la sixième qui avec qui vous pourrez chercher de vraies motivations

(ii) La substitution (FP-FS ou FS-FP) est possible avec une nuance de sens

21 - Dans l'exemple qui suit, si le FP (je vais demander) est remplaçable par un FS (je demanderai), la substitution provoque un glissement de sens, qui touche en particulier les deux traits de "validation" et d' " actualisation", qui auraient des valeurs distinctes (l'engagement du locuteur paraitrait moins ferme et un peu plus virtuel) :

(3) ESLO2_REP_14_C_5

Loc1 : en plus euh elle tu peux avoir super confiance hein puis elle est contente du coup [rire du locuteur] parce que ça ça lui évitera de la mettre sur Ebay et tout elle dit que ça la fait chier

Loc2 : eh je veux juste des informations hein pour le moment

Loc1 : hm mais ouais $\mathrm{hm}$ je vais demander euh à Pauline parce que hm j'ai vu la 
voiture que vite fait mais oui c' est une cinq portes c' est le euh hm deuxième modèle de Clio là celle qui est un petit peu arrondie là

En plus des trois valeurs principales habituellement identifiées, i.e. le prospectif (procès saisi dans sa phase préparatoire, cf. Jeanjean 1988, Wagner \& Pinchon 1991, Riegel et al. 1994, Wilmet $2007^{19}$ ), l'inaccompli (procès présenté en cours d'accomplissement et constituant souvent le cadre d'un autre procès, généralement ponctuel ; cf. Gosselin 1996), et le global (ou aoristique : le procès est perçu dans sa globalité ; cf. Wilmet 2007, pour le futur simple), nous avons identifié des emplois que l'on peut rapprocher de l'aspect progressif.

(i) Prospectif Cette valeur n'a été identifiée que pour le FP :

(7) ESLO2_CONF_1BGb_C_1

Loc1: et ces verriers vont émigrer dans toute l'Europe en apportant leur savoirfaire et ce savoir-faire qui va faire des allers-retours vu qu'on va le retrouver après en Italie parce qu'il y a bon y a tout un tas de contacts et je vais parler de l'un d'entre eux qui s'est établi à Orléans qui est donc Bernardo Perroto plus connu sous le nom de Bernard Perrot

(8) ESLO2_REP_01_02_C_11

Loc1 : quand il sera en troisième il sera dans la voix deux

$31-$ FP :

(9) ESLO1_ENT_003_C_17

Loc1: dès qu'il va rentrer dans le primaire je vais le mettre à l'école euh publique

(iii) Global Cette valeur concerne la majorité de nos exemples :

- FS : 
(10) ESLO2_REP_11_C_1

Loc1 : bah mets-lui ça on lui en remettra après si elle en reveut (iv) Progressif

Une dizaine d'occurrences se présentent, au FP seulement, avec des verbes comme durer, s'étendre, se prolonger, s'éliminer, en arriver à, continuer, etc. La combinaison entre un verbe de type duratif et le FP semble particulièrement propice à l'expression d'une valeur progressive pour un procès déjà engagé, dont l'accomplissement en cours est présenté comme se prolongeant dans l'avenir. ${ }^{21}$ :

(11) ESLO1_ENT_001_C_4

Loc1: euh la vente sous cellophane malheureusement va s'étendre puisque c'est dans les traditions maintenant

\subsection{Lien avec le présent}

Nous cherchons avec ce trait à vérifier l'une des hypothèses régulièrement proposées dans la littérature selon laquelle le FP exprimerait un procès en lien avec le présent (Fleischman 1983, Jeanjean 1988, Wagner \& Pichon 1991, Confais 1990, Wilmet 200722), alors que le FS servirait à exprimer des procès détachés du moment de l'énonciation. L'annotation exige une définition précise de la notion de lien qui semble avoir des acceptions relativement divergentes. Sera considéré ici comme lié au Présent tout procès qui se trouve soit déjà engagé (Je vais rentrer déjà chez moi (j'ai déjà fait quelques pas)), soit dans sa phase préparatoire (Je vais rentrer chez moi (je suis en train de ramasser mes affaires)), soit il s'agit d'un procès dont les conditions de réalisation se trouvent remplies dans $\mathrm{t}_{0}$ (C'est ce qu'on va manger ce soir (décision déjà prise); Elle va avoir un bébé (elle est déjà enceinte)).

(i) Procès lié au Présent

- FP :

(12) ESLO1_ENT_008_C_9

Loc1 : comment est-ce qu'on fait une omelette chez vous?

Loc2 : elle allait en faire une

Loc $1:$ ah c'est pourquoi je vous pose la question

Loc 2 : elle allait en faire une justement c'est ce qu'on va manger ce soir

$-\mathrm{FS}$

(13) ESLO2_CONF_5FLb_C_1

Loc1: et puis euh j'ai bien compris que il se fait tard et que euh comme en géographie d'ailleurs on lie toujours l'utile à l'agréable et euh lorsqu'on est géographe ruraliste et quand en plus on travaille sur la vigne et le vin généralement ça se termine toujours non pas en chanson mais dans des caves et donc avec force dégustation c'est ce que nous ferons ce soir voilà donc la géographie est très heureuse de vous accueillir pour ces travaux pratiques

39 (ii) Procès détaché

$40-$ FS :

(14) ESLO1_CONF_503_B_23

Loc1: je suis bien de votre avis plus on commencera tôt ce dressage et moins on pourra toujours on pourra de dispenser à ce niveau-là de chercher des motivations qui n'existent finalement pas oui n'est-ce pas hein c'est-à-dire que quand on commencera à apprendre l'anglais partout à huit ans eh bien vous o- vous organisez la actuel de ce qu'on appelle la la sixième qui avec qui vous pourrez chercher de vraies motivations 
$41-$ FP :

(15) ESLO1_ENT_009_C_27

Loc1 : ben alors qu'est-ce que ça fera ces jeunes-là quand les parents vont plus être

là?

\subsection{Proximité}

Il s'agit d'un trait relativement proche de la notion de lien, mais qui a été distingué en ce qu'il cherche à mesurer la distance, strictement chronologique, plus ou moins importante qui sépare le procès au futur de $t_{0}$. Là aussi, il est question de vérifier une hypothèse classique qui tente d'expliquer l'opposition FP/FS en termes de proximité avec la situation d'énonciation. L'annotation de la distance temporelle est délicate car elle pose la question du temps chronologique et du temps perçu dans les cadres de l'expérience. Nous avons cherché ici à considérer conjointement 1) des indices co-textuels explicites quand ils existaient (dans 4 ans vs ce soir) que l'on a mis en perspective 2) avec la situation d'énonciation, pour prendre en compte une possible variation de l'échelle en fonction du cadre d'expérience (à la rentrée prochaine peut avoir une valeur de proximité ou de distance temporelle selon la visée du locuteur, et l'analyse qualitative fondée sur la prise en compte d'un contexte interactionnel large permet généralement de statuer sur cette visée). La formulation "plutôt proche" vs "plutôt éloigné" reflète ce travail de va-et-vient entre indices co-textuels explicites et adaptation de l'échelle d'interpétation d'un contexte interactionnel large.

(i) Procès engagé Ce cas de figure n'a été identifié que pour le FP :

(16) ESLO2_ENT_1010_C_16

LOC1 : donc je me suis dit ah j'ai peut-être pas choisi le le bon secteur

LOC2 : hm hm le bon filon

LOC 1 : et en fait c'est vraiment ça quoi c'est à dire que là au bout au bout de quatre ans on est plus que seize mille

LOC2 : en quatre ans ça a été d'accord ouais ouais ouais donc s-

LOC 1 : seize mille et euh c'est que 1 - voilà hein donc on est dans l'ère où ça va encore continuer de de réduire

$45-$ FP

(17) ESLO2_ENT_1010_C_17

Loc1 : en fait le plan va avoir lieu là dans peu de temps donc ça sera trop tard

(18) ESLO2_CONF_1074_C_1

Loc1 : l'après-midi va va durer euh un certain temps hein puisque nous avons prévu euh cinq euh interventions euh vous avez la possibilité euh bien sûr d'aller prendre un café euh des petits gâteaux pour vous restaurer et puis vous sortir un petit peu bon $\mathrm{hm}$ voilà entre deux entre deux conférences on va dire euh on fera pas vraiment de pauses euh complètes qui risquent de faire dériver le le le temps

(iii) Procès plutôt éloigné

$48-$ FP :

(19) ESLO1_CONF_504_A_22

Loc1: vous savez que ces programmes dans l'idée de la commission sont quadriennaux tous les quatre ans ça ne veut pas dire que dans quatre ans on va déchirer tout ça et puis inventer d'autres choses n'est-ce pas 
$49-$ FS :

(20) ESLO1_REPAS_272_B_18

Loc1 : ils ont eu une chance en soixante-huit euh ça se reproduira pas avant avant vingt ou trente ans

\subsection{Validation}

Il n'a pas été ici question de distinguer toutes les valeurs modales épistémiques (possible, probable, certain, contingent, etc.), mais uniquement deux valeurs essentielles : le certain et le non certain, afin de vérifier l'une des hypothèses quelquefois proposées selon laquelle le FS exprimerait un procès moins certain que le FP (Confais, $1990: 284)$. À ces deux valeurs essentielles, trois autres valeurs ont dû être ajoutées : non pertinent, vrai (pour le futur historique), et engagement (direction d'ajustement allant de l'énoncé au monde, cf. Gosselin 2010).

51 (i) Procès présenté comme certain

$52-$ FP :

(21) ESLO1_ENT_003

JR : et est-ce que vous avez des projets pour les vacances d'été?

DJ : ah pour les vacances d'été je vais aller euh je vais aller comme tous les ans au Guilvinec qui est un petit port euh breton où $\mathrm{y}$ a beaucoup plus de d'autochtones que de touristes

$53-$ FS :

(22) ESLO2_CONF_4CPMEb_C_8

Loc1 : euh ce roman a reçu le sixième prix Vargas Llosa de roman en Espagne il sera publié à la rentrée prochaine

(ii) Procès présenté comme non certain

$55-$ FS :

(23) ESLO2_ENT_1010

ch_CD 2 : à voir quoi il est prévu pour quand?

GK : nous on l- il est prévu pour deux mille treize je crois

ch_CD d'accord oui c'est [inintelligible]

GK [inspiration] mise en service donc y a encore euh trois trois bonnes années de [bruit de bouche] d' ennui [rire du locuteur] pour se garer

ch_CD $2: 0: 08: 44$ [rire du locuteur] c'est ça c'est ça

GK $11: 0: 08: 46$ mais euh sinon euh ouais mais je pense qu'on sera plus là quand le tram euh lui sera là

$56-$ FP :

(24) ESLO2_REP_11_C_3

Loc1 : je vais te le couper

Loc2 : je crois pas que je vais manger les deux tranches

Loc1 : mais alors ah bah si ça fait trop je peux en enlever là hein

57 (iii) Procès présenté comme vrai (futur historique)

$58-$ FP :

(25) ESLO2_CONF_1BGb_C_5

Loc1: Bernard Perrot c'est un Orléanais il va laisser des tra- un certain nombre de traces dans Orléans dont les traces les plus importantes sont dans les roses des cathédrales n- nord euh des transepts nord et sud de la cathédrale d'Orléans comme euh nous avons pu le montrer récemment 
$59-$ FS :

\section{(26) ESLO2_CONF_4CPMEb}

Loc1 : le récit se clôt là où il avait commencé à la frontière franc- franco-espagnole en Hendaye l'endroit où le père de Jacques avait disparu et où Jacques mourra aussi incapable de dépasser les clivages linguistiques et culturels que sa mère avait vainement essayé de supprimer en lui

$61-$ FP :

(27) ESLO2_REP_11_C_2

Loc1 : tu te souviens dans la voiture je te disais j'avais soif

Loc2 : ah oui c'est vrai je vais t'en donner tout de suite moi aussi j'ai soif

$62-$ FS :

(28) ESLO1_CONF_503_B_2

Loc1: on serait tenté de croire que l'apprentissage d'une langue étrangère c'est quelque chose d'assez semblable d'assez allogue euh d'assez parallèle à l'apprentissage de la langue maternelle euh vous me direz ce que vous en pensez pour ma part je vous le dirai aussi mais enfin euh là-dessus la discussion reste ouverte euh

\subsection{Actualisation}

63 Avec ce trait, il est question de savoir si le procès est présenté comme actuel (dans le sens d'occurrent) ou plus virtuel. En plus des cas de non pertinence, où le procès n'exprime aucune valeur sur l'échelle d'actualisation, deux possibilités sont distinguées.

(i) Procès présenté comme virtuel

$65-$ FS :

(29) ESLO1_ENT_009:

G258 : on pense pas bon ben c'est ma taille on dit oui ou si

on leur dit si ça ne va pas vous reviendrez

$66-$ FP :

(30) ESLO1_ENT_009_C_28

Loc1 : et sa fille ben sa fille elle a carrément dit à son père dis-donc moi c'est trop tenu je vais pas faire dactylo je veux mon temps je veux me promener bon ben la fille maintenant elle est elle est toujours célibataire et elle compte trop sur papa maman c'est pas ça qui va nous sauver

67 (ii) Procès présenté comme actuel

$68-$ FS :

(31) ESLO2_CONF_5FLb_C_1

Loc1: et puis euh j'ai bien compris que il se fait tard et que euh comme en géographie d'ailleurs on lie toujours l'utile à l'agréable et euh lorsqu'on est géographe ruraliste et quand en plus on travaille sur la vigne et le vin généralement ça se termine toujours non pas en chanson mais dans des caves et donc avec force dégustation c'est ce que nous ferons ce soir voilà donc la géographie est très heureuse de vous accueillir pour ces travaux pratiques

$69-\mathrm{FP}$

(32) ESLO1_ENT_003_C_17

Loc1: dès qu'il va rentrer dans le primaire je vais le mettre à l'école euh publique publique 


\section{Analyse quantitative : quelques hypothèses explicatives}

\subsection{Tendances générales}

Récapitulées dans le tableau 2, les premières données quantitatives montrent que si le nombre d'occurrences du futur (en tant que catégorie générique regroupant les deux tiroirs verbaux étudiés) reste remarquablement stable d'ESLO1 à ESLO2, la répartition interne entre le FS et le FP s'inverse totalement ${ }^{24}$, comme on peut le visualiser sur le graphique suivant :

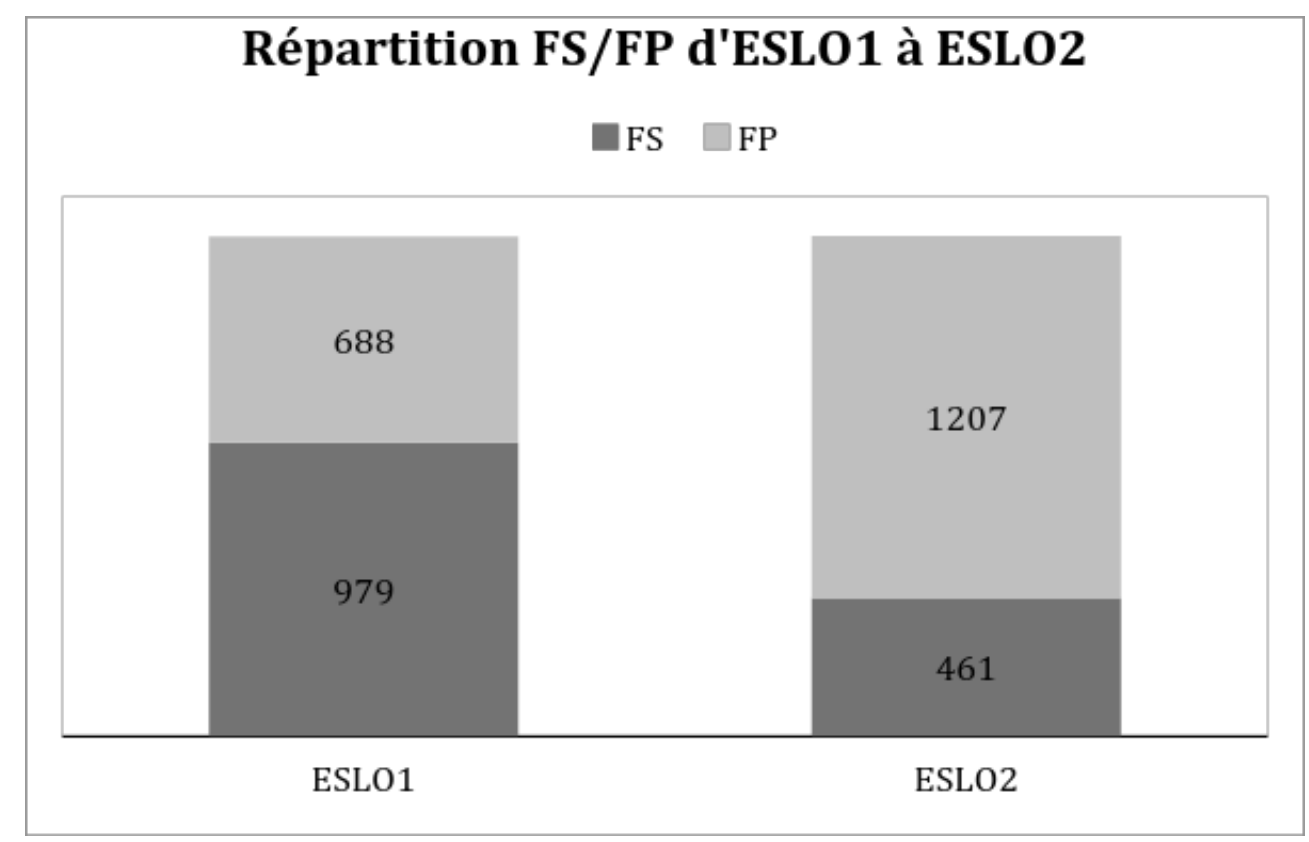

Figure 2 : Nombre d'occurrences des formes du futur simple (fs) et du futur périphrastique (fp) : tendances en micro-diachronie (ESLO1 - ESLO2)

Un examen plus précis de ces tendances en fonction des types d'emplois, opposant les emplois purement chronologiques aux emplois contenant un trait modal (typés aussi bien « $\mathrm{m} »$ que « $\mathrm{fm} »)^{25}$, montre, en ce qui concerne le FP, une progression importante aussi bien dans ses emplois temporels que dans ses emplois modaux, et dans des proportions assez comparables (aux alentours de $+75 \%$ ), ce qui nous incite à parler de progression $a$ priori homogène ${ }^{26}$ : 


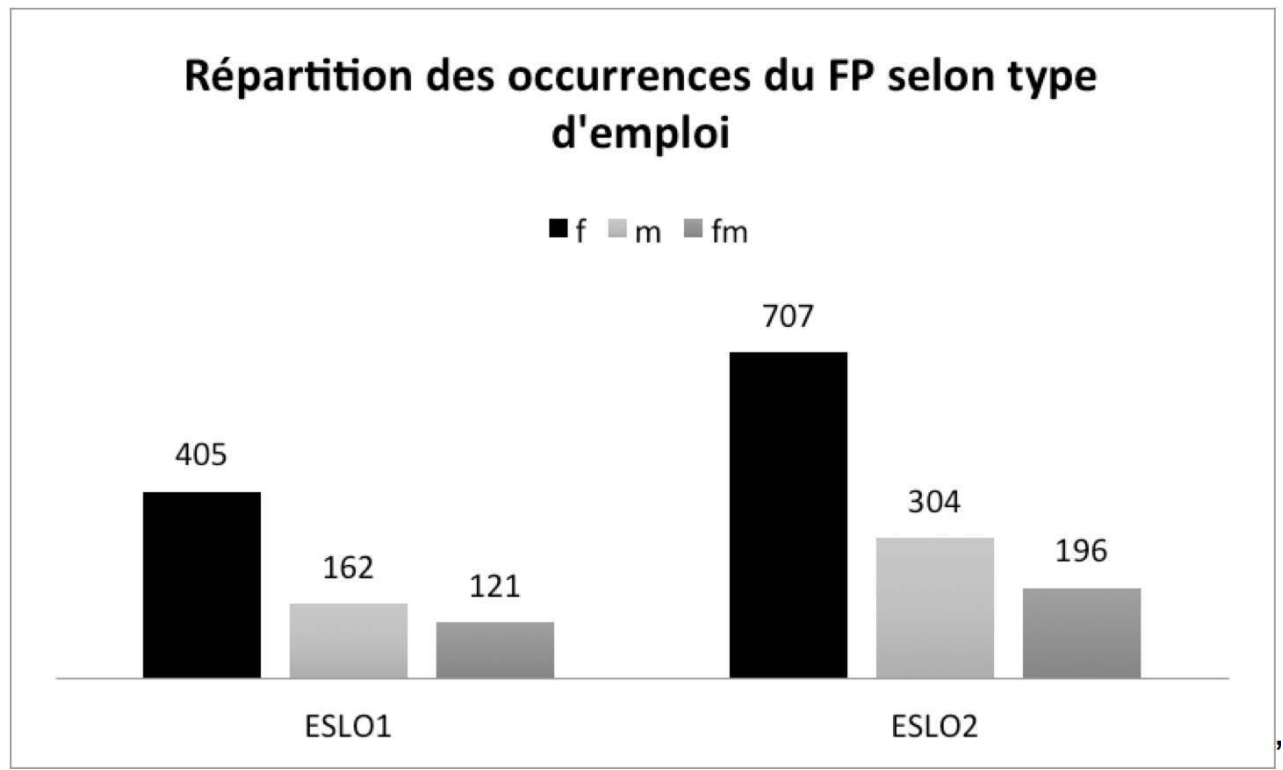

Figure 3 : Répartition des emplois du FP en nombre d'occurrences

72 En ce qui concerne le FS, en revanche, on observe que le recul est particulièrement net pour les emplois modaux, qui chutent d'environ $80 \%$, là où les emplois temporels ne reculent que d'environ $30 \%$ :

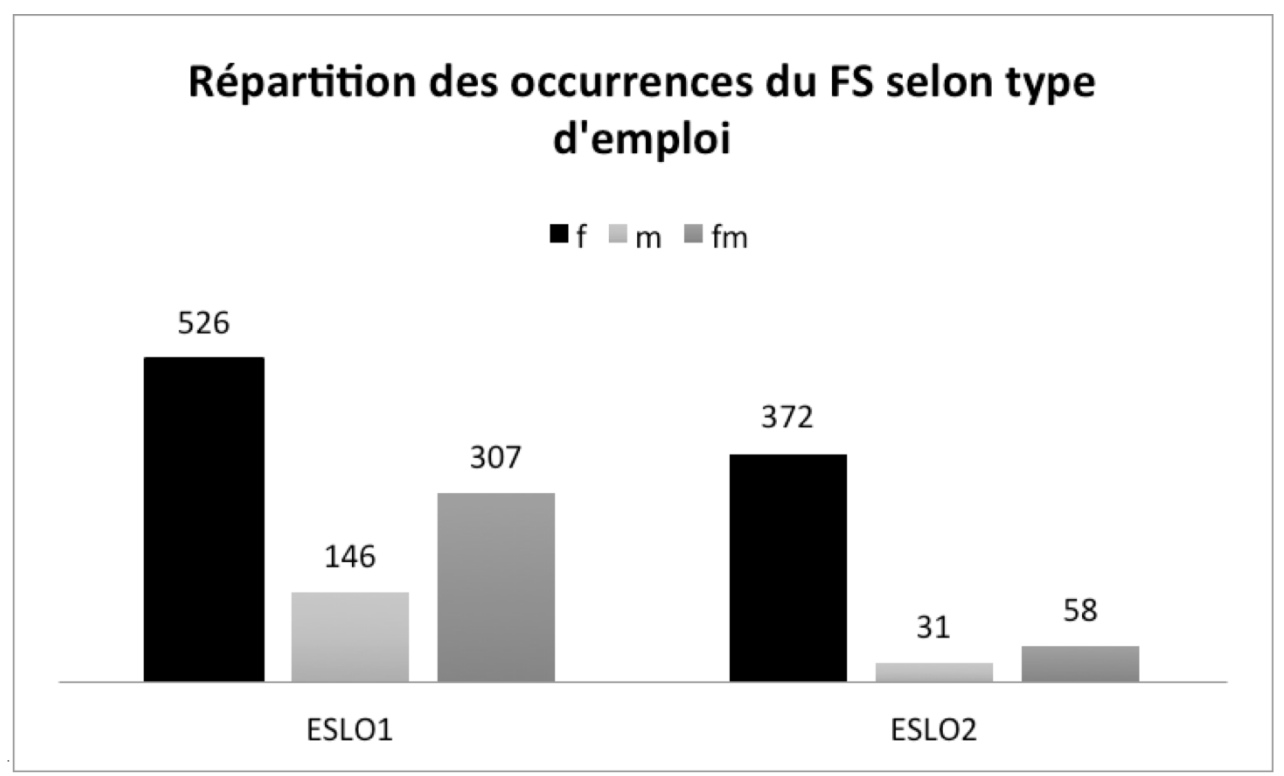

Figure 4 : Répartition des emplois du FS en nombre d'occurrences

Ces tendances générales montrent d'emblée une certaine dissymétrie entre la forte progression des emplois temporels du FP et le recul plus modeste des emplois temporels du FS. Nous nous proposons ici, à l'image de ce que nous avions déjà entrepris pour les emplois modaux, dont la baisse sensible avait d'emblée retenu notre attention (cf. Abouda \& Skrovec 2015) d'examiner l'évolution micro-diachronique des emplois temporels, en fonction des différents traits annotés. 


\subsection{FS/FP en micro-diachronie : valeurs temporelle et aspectuelle}

74 distribution des formes annotées « $\mathrm{f}$ » et le recul du FS face au FP. Globalement, la répartition décrite dans la littérature entre un $\mathrm{FP}$ exprimant des procès proches, liés et présentés comme certains et un FS exprimant des procès détachés, éloignés de $t_{0}$ et incertains, se maintient. Ainsi, si l'on considère pour le trait « proximité » l'ensemble des occurrences exprimant un procès déjà engagé ou plutôt proche, on observe dans ESLO1 que plus de $88 \%$ sont des FP et seulement $12 \%$ sont des FS ; dans ESLO2, cette répartition évolue, mais la tendance reste la même: $79 \%$ de FP et $21 \%$ de FS (cf. nombre d'occurrences, tableau 4). Par ailleurs, le trait opposé, celui de l'éloignement temporel, est assumé aussi bien dans ESLO1 que dans ESLO2 par le FS majoritairement.

\begin{tabular}{|l|l|l|l|l|}
\hline \multicolumn{2}{|l|}{ Proximité avec $\mathrm{t}_{0}$} & FP & FS & Total \\
\hline \multirow{2}{*}{ ESLO1 } & déjà engagé ou plutôt proche & 119 & 16 & 135 \\
\cline { 2 - 5 } & plutôt éloigné & 5 & 66 & 71 \\
\hline \multirow{2}{*}{ ESLO2 } & déjà engagé ou plutôt proche & 106 & 29 & 135 \\
\cline { 2 - 5 } & plutôt éloigné & 0 & 47 & 47 \\
\hline
\end{tabular}

Tableau 4 : Trait proximité avec $t_{0}$, en nombre d'occurrences

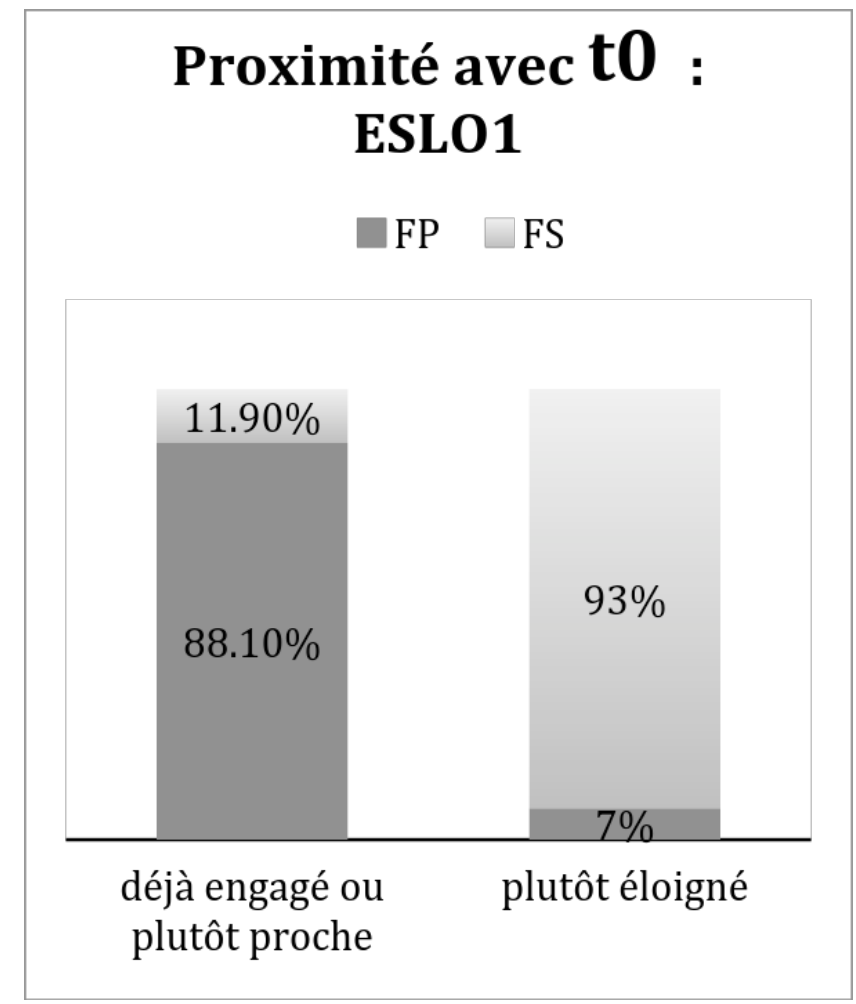




\section{Proximité avec t0 :} ESLO2

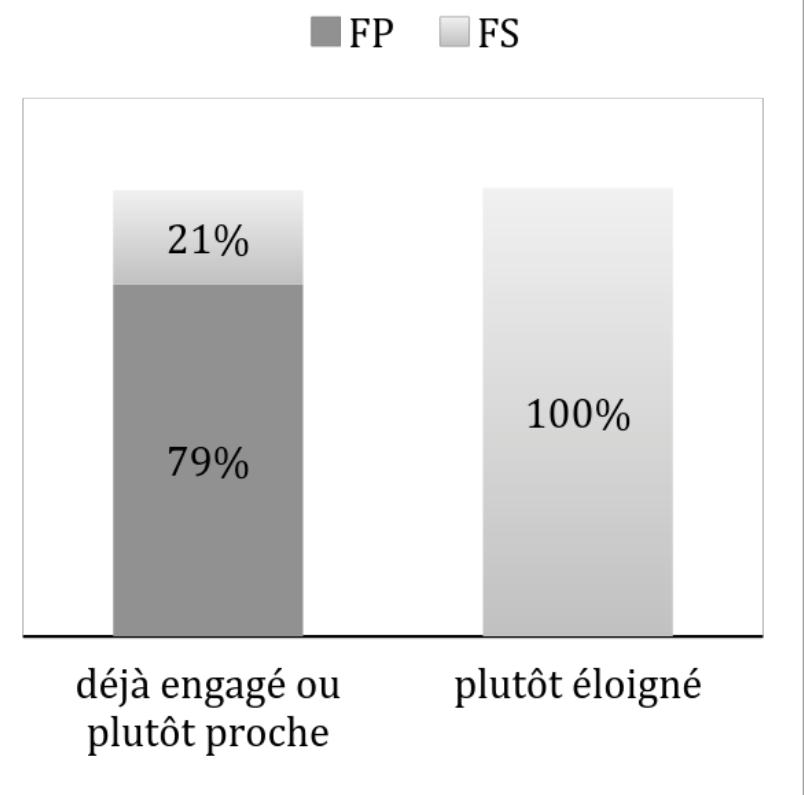

Figures 5 et 6 : Trait proximité avec $t_{0}$ en pourcentages dans ESLO1 et ESLO2

En outre, le FP conserve des contextes d'emplois spécifiques dans lesquels il se maintient en exclusivité comme l'aspect prospectif (34) qui n'est jamais saisi au FS. Il maintient également sa prévalence dans d'autres emplois comme l'engagement énonciatif (33): il est plus souvent sélectionné pour cette valeur, selon une tendance qui se confirme en micro-diachronie (tableau 5 , figure 7).

(33) ESLO2_CONF_1BGb_C_1

Loc1 : et ces verriers vont émigrer dans toute l'Europe en apportant leur savoirfaire et ce savoir-faire qui va faire des allers-retours vu qu'on va le retrouver après en Italie parce qu'il y a bon y a tout un tas de contacts et je vais parler de l'un d'entre eux qui s'est établi à Orléans qui est donc Bernardo Perroto plus connu sous le nom de Bernard Perrot

(34) ESLO2_REP_11_C_2

Loc1 : tu te souviens dans la voiture je te disais j'avais soif

Loc2 : ah oui c'est vrai je vais t'en donner tout de suite moi aussi j'ai soif

\begin{tabular}{|l|l|l|l|}
\hline Engagement énonciatif & FP & FS & Total \\
\hline ESLO1 & 28 & 15 & 43 \\
\hline ESLO2 & 49 & 11 & 60 \\
\hline
\end{tabular}

Tableau 5 : Engagement énonciatif en nombre

d'occurrences 


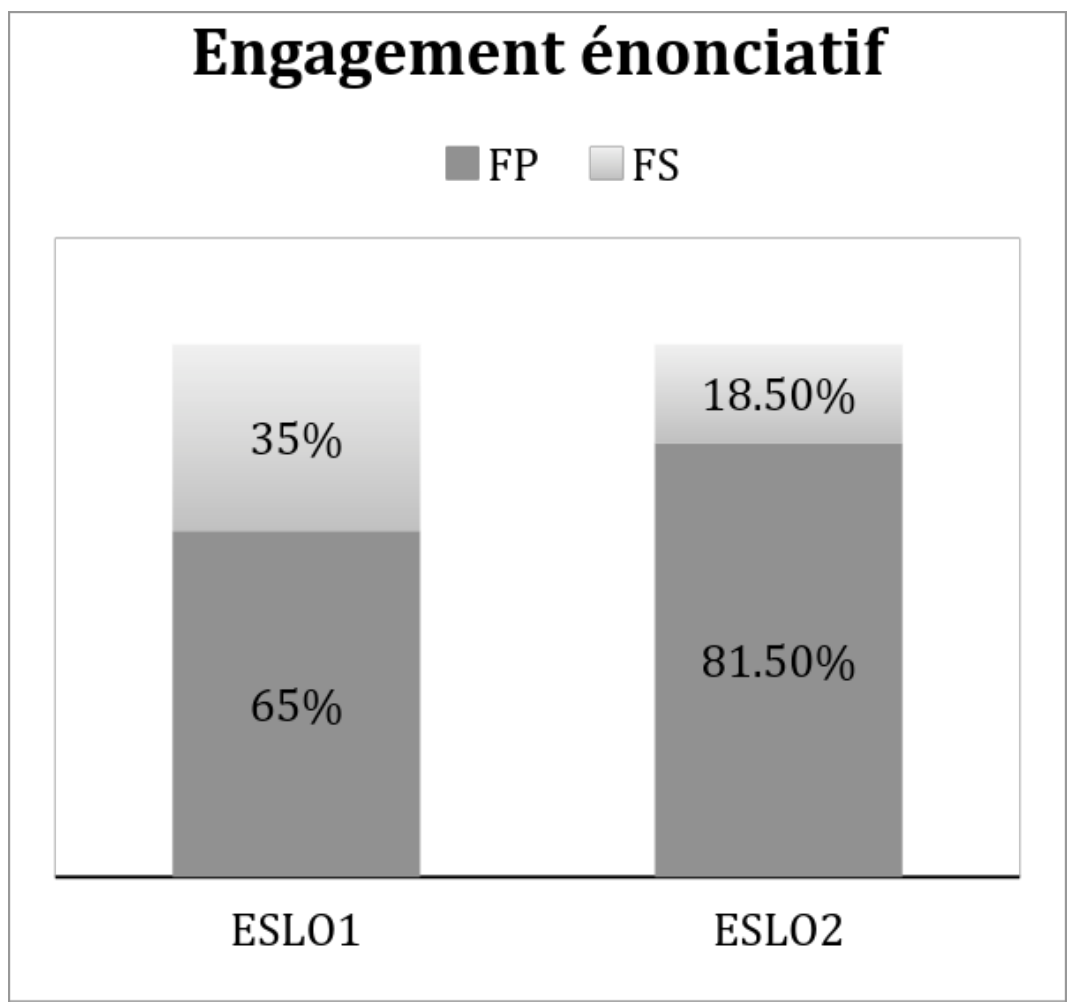

Figure 7 : Engagement énonciatif en pourcentages

Par ailleurs, il concurrence le FS par une augmentation de sa capacité à occuper des emplois d'habitude assumés par le FS, comme l'aspect global par exemple :

(35) ESLO1_ENT_003_C_17

Loc1 : dès qu'il va rentrer dans le primaire je vais le mettre à l'école euh publique

En effet, si l'on considère la répartition des formes pour exprimer cette valeur, la concurrence est visible entre ESLO1 et ESLO2. Les chiffres ci-dessous (tableau 6, figure 8) témoignent du fait que le FP est plus souvent qu'avant convoqué pour exprimer l'aspect global, au détriment du FS, qui l'est moins souvent dans ESLO2 que dans ESLO1.

\begin{tabular}{|l|l|l|l|}
\hline Aspect global & FP & FS & total \\
\hline ESLO1 & 110 & 149 & 259 \\
\hline ESLO2 & 136 & 113 & 249 \\
\hline
\end{tabular}

Tableau 6 : Aspect global en nombre d'occurrences 


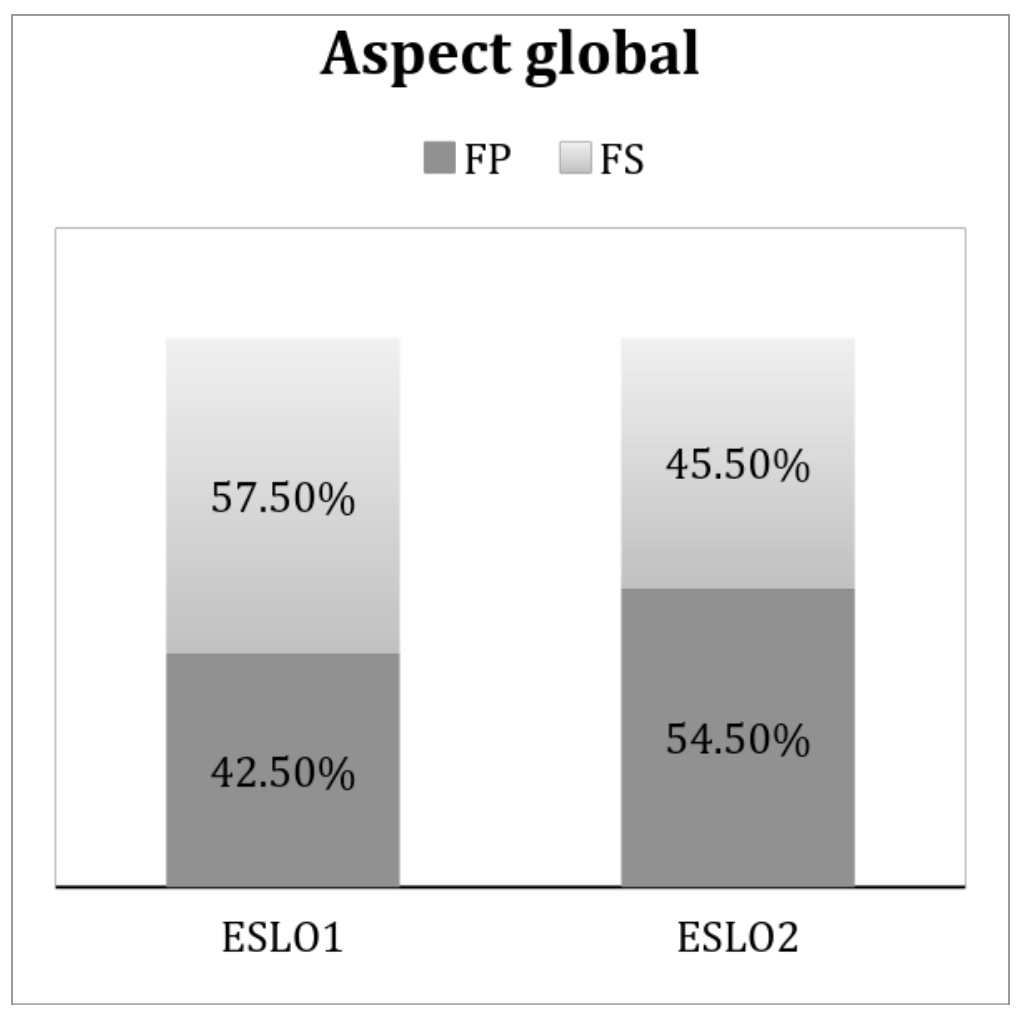

Figure 8 : Aspect global en pourcentages

Il semble également que le FP, tout en n'étant pas concurrencé dans ses valeurs typiques comme la proximité ou le lien avec le présent, montre diachroniquement une plus grande capacité à neutraliser ces traits dans d'autres contextes et sur un nombre d'occurrences non négligeable. L'essor quantitatif du FP reposerait donc sur plusieurs tendances observables : une capacité à garder l'avantage sur ses valeurs typiques, tout en étant plus polyvalent puisque plus fréquemment impliqué dans des emplois où cette typicité n'est plus pertinente, comme le montrent le tableau 7 et la figure 9. En outre, il est plus fréquemment sélectionné pour des emplois habituellement pris en charge par le FS (aspect global).

\begin{tabular}{|l|l|l|}
\hline FP & ESLO1 & ESLO2 \\
\hline Trait Lien avec le présent : non pertinent & 3 & 33 \\
\hline Trait Proximité avec T0 : non pertinent & 34 & 67 \\
\hline Nombre total de FP annotés & 158 & 173 \\
\hline
\end{tabular}

Tableau 7 : Progression de la valeur non pertinent pour les traits Lien et Proximité d'ESLO1 à ESLO2 en nombre d'occurrences pour le FP 


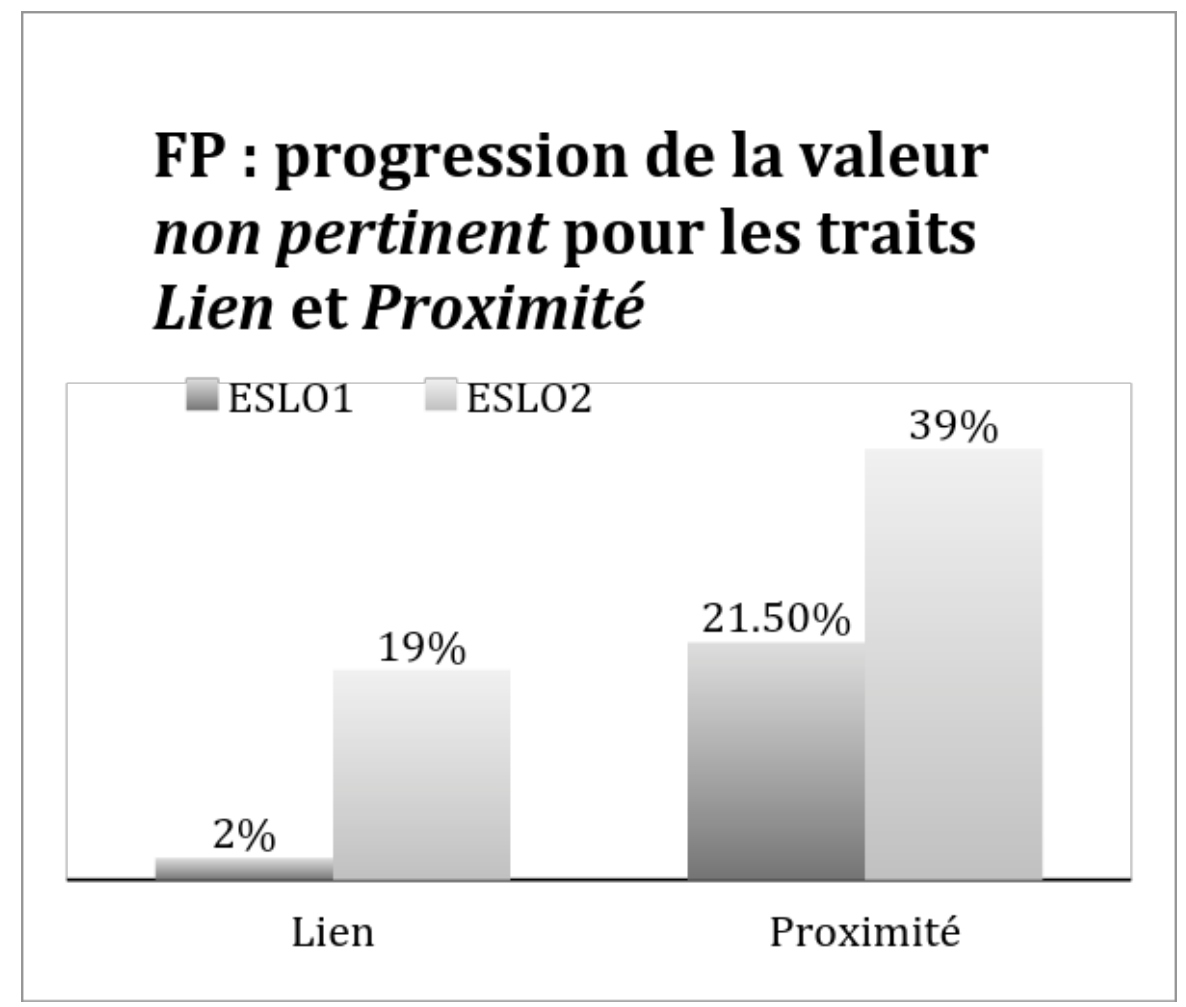

Figure 9 : Progression de la valeur non pertinent pour les traits Lien et Proximité d'ESLO1 à ESLO2 en pourcentages pour le FP

De son côté le FS, qui recule quantitativement, se maintient en se spécialisant qualitativement dans ses propriétés typiques. Il est ainsi plus souvent sélectionné pour référer à un évènement détaché du présent de l'énonciation (cf. 36, tableau 8, figure 10), où les éléments ne sont pas réunis dans $t_{0}$ pour que le procès soit validé, ou à un évènement plutôt éloigné comme on l'a vu précédemment (tableau 4, figures 5 et 6 ).

(36) ESLO2_ENT_1002_C_15

on est un peu dans l'urgence donc euh bon pas dans l'urgence médicale on est dans une urgence quand même quand il y a une demande et que on sait que ça va faciliter euh oui faut répondre quoi $\mathrm{hm}$ la vie des personnes et qu'on sait qu'actuellement euh dans ce qui vit il est en souffrance on peut pas se permettre de leur dire bah je vous répondrai dans quinze jours donc euh ouais $\mathrm{hm} \mathrm{hm} \mathrm{hm}$ faut trouver des solutions rapides

\begin{tabular}{|l|l|l|l|l|l|}
\hline FP & ESLO1 & ESLO2 & FS & ESLO1 & ESLO2 \\
\hline lié & 122 & 99 & lié & 29 & 20 \\
\hline détaché & 33 & 41 & détaché & 116 & 92 \\
\hline non pertinent & 3 & 33 & non pertinent & 4 & 3 \\
\hline
\end{tabular}

Tableau 8: Valeurs du FS pour le trait Lien d'ESLO1 à ESLO2 en nombre d'occurrences 


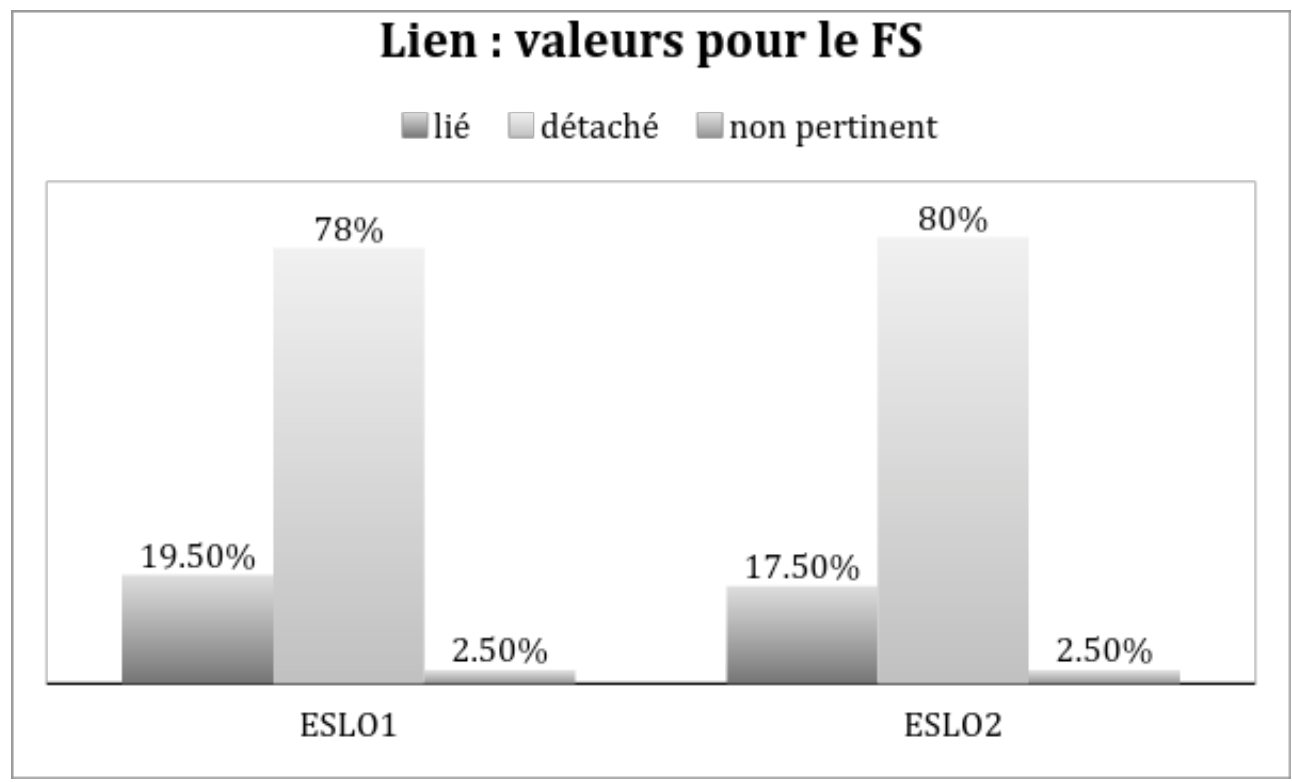

Figure 10 : Valeurs du FS pour le trait Lien d'ESLO1 à ESLO2 en pourcentages

Enfin, le test de substituabilité semble confirmer la tendance du FS à se spécialiser dans ces emplois. Le tableau 9 et la figure 11 ci-dessous montrent en effet que les cas de FS sont moins souvent substituables par un FP dans ESLO2 que dans ESLO1 (ce qui n'est pas le cas pour le FP). Ainsi, si l'on corrèle ce résultat au recul quantitatif du FS, l'hypothèse d'une spécialisation du FS semble confortée ${ }^{27}$.

\begin{tabular}{|l|l|l|}
\hline FS & ESLO1 & ESLO2 \\
\hline oui & 92 & 17 \\
\hline nua & 29 & 41 \\
\hline inad & 82 & 57 \\
\hline & 203 & 115 \\
\hline
\end{tabular}

Tableau 9 : Substituabilité du FS par un FP d'ESLO1 à ESLO2 


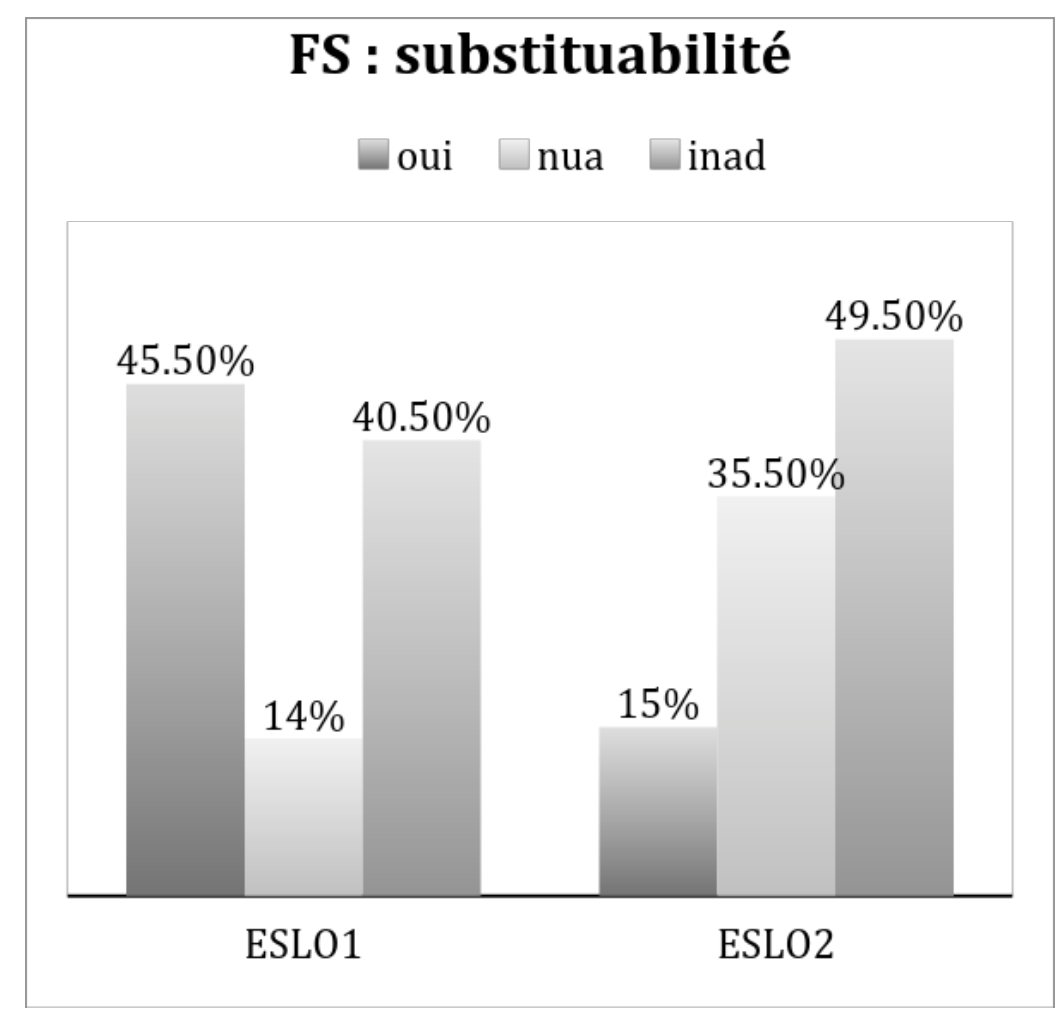

Figure 11 : Substituabilité du FS par un FP d'ESLO1 à ESLO2

\section{Conclusion}

L'exploitation des propriétés sémantiques annotées dans ce corpus constitué pour une analyse micro-diachronique nous éclaire sur le type de rapport à l'œuvre dans l'évolution des deux formes du futur en français moderne. Elle permet en effet d'entrevoir l'existence d'un rapport complexe à la fois de concurrence et de complémentarité entre le FP et le FS, selon une tendance générale similaire à celle observée pour les valeurs modales (cf. Abouda \& Skrovec, 2015). Pour les emplois de futurité, l'essor quantitatif du FP face au FS mis en évidence par une première couche d'annotation montre que le FS est bien concurrencé par le FP, même si cette concurrence n'est pas aussi forte que pour les emplois modaux. Un examen plus précis des types d'emplois permet d'en savoir un peu plus sur la nature de cette concurrence. Ainsi, on a pu observer que le FP non seulement se maintient quantitativement dans ses contextes d'emplois spécifiques (lien avec le présent, proximité avec $t_{0}$, valeurs aspectuelles telles que le prospectif, ainsi que l'expression de l'engagement énonciatif) mais aussi qu'il concurrence le FS par une augmentation de sa capacité à occuper des emplois d'habitude assumés par ce dernier, comme l'aspect global. On assiste également à une augmentation des cas de FP pour lesquels on note une perte de spécificité, en particulier le lien avec le présent et la proximité avec $t_{0}$ puisqu'on compte davantage de cas de FP catégorisés comme non pertinents dans ESLO2 que dans ESLO1. Face à cette concurrence, le FS semble se maintenir en se spécialisant qualitativement dans ses propriétés typiques comme l'expression du détachement par rapport à la situation d'énonciation par exemple, ce qui instaure une relation de complémentarité entre les deux tiroirs verbaux pour des valeurs comme le lien avec le présent et la proximité avec $t_{0}$, même si le FS est en recul face au FP. 


\section{BIBLIOGRAPHIE}

Abouda, L. (2015). Syntaxe et Sémantique en corpus. Du temps et de la modalité en français oral, Habilitation à Diriger des Recherches, Université d'Orléans.

Abouda, L. \& Baude, O. (2007). «Constituer et exploiter un grand corpus oral : choix et enjeux théoriques. Le cas des Eslo ", in F. Rastier et M. Ballabriga (dir.), Corpus en Lettres et Sciences sociales. Des documents numériques à l'interprétation, Actes du XXVIIe Colloque d'Albi « Langages et Signification »,161-168.

Abouda, L. \& Skrovec, M. (2015). « Du rapport entre formes synthétique et analytique du futur. Étude de la variable modale dans un corpus oral micro-diachronique ", Revue de Sémantique et Pragmatique, 38, 35-57.

Arrivé, M, Gadet, F., Galmiche, M. (1986). La Grammaire d'aujourd'hui. Guide alphabétique de linguistique française, Flammarion.

Barcelo, G. J. (2007). « Le(s) futur(s) dans les langues romanes : évolution linéaire ou cyclique ? ", Sémantique et diachronie du système verbal français, Cahiers Chronos, 16, Amsterdam, New York, NY, 47-62.

Biber, D. (2009). « Corpus-Based and Corpus-driven Analyses of Language Variation and Use » in : Bernd Heine and Heiko Narrog (ed.), The Oxford Handbook of Linguistic Analysis, Oxford University Press.

Bilger, M. (2001). « Retour sur le futur dans les corpus de français parlé », RSFP, 16, Université de Provence, 177-189.

Bybee, J.L., Pagliuca, W. \& Perkins, R.D. (1991). « Back to the future », in. Traugott, E.C. \& Heine, B. (eds). Approaches to the grammaticalization, II, Amsterdam/Philadephia, John Benjamins Publishing Company, 17-58.

Celle, A. (2005). « The French future tense and English will as markers of epistemic modality », Languages in Contrast, 5,181-218.

Collier, M. (2013). « The aller Perfect », in Labeau, E., Bres, J. (ed.), Evolution in romance verbal systems, Peter Lang, 203-220.

Confais, J.-P. (1990). Temps, Mode, Aspect, Toulouse, Presses Universitaires de Mirail.

Desahaies, D. \& Laforge, E. (1981). « Le futur simple et le futur proche dans le français parlé dans la ville de Québec ». Langues et Linguistique, 7, 21-37.

Emirkanian, L. ; Sankoff, D. (1986). « Le futur simple et le futur périphrastique dans le français parlé » in Morphosyntaxe des langues romanes, Actes du XVIIe Colloque international de linguistique et de philologie romanes, Aix-en-Provence, 397-407.

Fleischman, S. (1982). The future in thought and language, New York, Cambridge University Press.

Fleischman, S. (1983). « From pragmatics to grammar : diachronic reflections on complex pasts and futures in Romance ", Lingua, 60, 183-214. 
Fleury, S. \& Branca, S. (2010). «Une expérience de collaboration entre linguiste et spécialiste de TAL : L'exploitation du corpus CFPP 2000 en vue d'un travail sur l'alternance Futur simple / Futur périphrastique ", Cahiers AFLS, Volume 16(1).

Gosselin, L. (1996). Sémantique de la référence temporelle en français. Louvain-la-Neuve, Duculot. Gosselin, L. (2005). Temporalité et modalité, Bruxelles, Duculot-de Boeck, col. Recherches. Heiden, S., Mague, J-P., Pincemin, B. (2010). « TXM : Une plateforme logicielle open-source pour la textométrie - conception et développement ", in I. C. Sergio Bolasco (Ed.), Proc. of 10th International Conference on the Statistical Analysis of Textual Data - JADT 2010, vol. 2, 1021-1032, Edizioni Universitarie di Lettere Economia Diritto, Roma, Italy.

Heiden, S. (2010). « The TXM Platform : Building Open-Source Textual Analysis Software Compatible with the TEI Encoding Scheme », in K. I. Ryo Otoguro (Ed.), 24th Pacific Asia Conference on Language, Information and Computation - PACLIC24, 389-398, Institute for Digital Enhancement of Cognitive Development, Waseda University, Sendai, Japan.

Jeanjean, C. (1988). « Le futur simple et le futur périphrastique en français parlé : Étude distributionnelle » in C. Blanche-Benveniste et al. Grammaire et histoire de la grammaire : Hommage à la mémoire de Jean Stéfanini. PU de Provence, 235-257.

Lansari, L. (2009). Linguistique contrastive et traduction. Les périphrases verbales aller + infinitif et be going to, Ophrys.

Laurendeau, P. (2000). «L'alternance futur simple/futur périphrastique : une hypothèse modale ", Verbum, tome 22, n 3, PU de Nancy, 277-292.

Maingueneau, D. (1994). L'énonciation en linguistique française, 2e édition (1999), Hachette.

Mauger, G. (1968). Grammaire pratique du français d'aujourd'hui. Paris, Hachette.

Mullineaux, L.A. \& Blanc, M. H. A. (1982). « The problem of classifying the population sample in the socio linguistic survey of Orléans (1969) in terms of socio-economic, social and educational categories ", Review of Applied Linguistics, 55, 3-37.

Rocchetti, A. (2004), « La syntaxe du futur et du conditionnel dans les langues romanes : le futur après "si" et le futur dans le passé ", in Luquet, G., Rocchetti, A. (ed.), Regards sur la syntaxe historique des langues romanes. Paris : Presses de l'Université Paris-III, 67-106.

Wagner, R.-L. \& Pinchon, J. (1991). Grammaire du Français Classique et Moderne, Hachette Wilmet, M. (2007). Grammaire critique du français, 4e édition, de Boeck \& Larcier, Bruxelles.

\section{NOTES}

1. Les Enquêtes Sociolinguistiques à Orléans est un corpus d'enregistrements audio constitué à des fins linguistiques et didactiques qui vise à réaliser le portrait sonore de la ville d'Orléans par ses habitants. Voir pour une présentation des ESLO Abouda \& Baude (2007) et http://eslo.humanum.fr/

2. On passe ainsi de $42 \%$ de FP pour $58 \%$ de FS à $72 \%$ de FP pour $28 \%$ de FS.

3. Pour une présentation détaillée des genres interactionnels représentés dans cette étude, on peut consulter : http://eslo.huma-num.fr/index.php/pagecorpus/pagepresentationcorpus

4. Nous avons repris les catégories de l'échelle Alix Mullineaux (échelle AM, cf. Mullineaux \& Blanc 1982), utilisés pour les entretiens ESLO1, en les adaptant au cas par cas aux profils des locuteurs d'ESLO2. 
5. Heiden \& al. (2010) et Heiden (2010). Voir également http://textometrie.ens-lyon.fr/

6. http://www.cis.uni-muenchen.de/ schmid/tools/TreeTagger/

7. CQL, acronyme pour Corpus Query Language, est le langage d'expression de requêtes utilisé pour interroger les corpus sous TXM. La syntaxe de la requête utilisée pour identifier les FP est la suivante : [frlemma ="aller"\&frpos ="VER :pres"][]\{0,5\}[frpos ="VER :infi"] et pour le FS : [frpos ="VER :futu"]. Nous avons donc cherché toutes les occurrences du verbe aller au présent suivies d'un infinitif dans un contexte de 5 mots après aller.

8. Cette distribution s'éclaire quelquefois mieux lorsqu'on croise ces informations avec des métadonnées relatives au genre interactionnel et à l'activité de communication en cours. Par exemple, le futur périphrastique historique n'apparait dans notre corpus que dans ESLO2 : loin de correspondre à une quelconque évolution micro-diachronique, cet usage du FP s'explique par le fait que l'une des conférences d'ESLO2 est une conférence d'Histoire.

9. Après une première phase d'annotation commune, lors de laquelle les étiquettes forgées ont été stabilisées, chaque expert a annoté la totalité des occurrences concernées, avant de procéder à une comparaison systématique entre les deux annotations. Les cas de désaccord ont été systématiquement discutés pour arriver à un consensus.

10. Encore que l'extension exacte de la classe des emplois temporels et celle des emplois modaux varie selon les auteurs. Pour nous, l'emploi temporel est un emploi qui situe le procès dans un moment ultérieur à un repère référentiel, qui est généralement le moment de l'énonciation, mais qui peut aussi correspondre à un repère distinct, comme c'est le cas pour le futur historique :

ESLO2_CONF_4CPMEb_C_11:

le récit se clôt là où il avait commencé à la frontière franc- franco-espagnole en Hendaye l'endroit où le père de Jacques avait disparu et où Jacques mourra aussi incapable de dépasser les clivages linguistiques et culturels que sa mère avait vainement essayé de supprimer en lui ou encore pour le futur relatif :

ESLO2_CONF_5FLb_C_3 :

Platon mais aussi d'autres évoquent le symposium grec où les convives se passent un cratère où dans lequel il y a de l'eau et du vin et ils font l'éloge de l'amour mais pas seulement n'est-ce pas lors de ces banquets et en quelque sorte ils se lancent des défis et ils essayent de finalement de de faire le l'éloge qui sera le le plus apprécié par tous hein

11. Pour d'autres exemples commentés de la catégorie fm et une discussion sur l'opposition entre futurité et modalité, voir Abouda \& Skrovec (2015).

12. La méthodologie choisie ici, qui, se basant sur l'exploration exhaustive d'un corpus, impose de considérer les données dans toute leur réalité et complexité, et donc d'intégrer des emplois hybrides qui auraient sans doute été écartés s'il était simplement question de glaner des occurrences à partir de différentes sources pour illustrer des catégories préétablies.

13. Abouda \& Skrovec (2015).

14. Les emplois mixtes n'ont pour le moment pas fait l'objet d'un étiquetage systématique. Un arbitrage a dû être fait pour donner la priorité à un travail d'annotation permettant d'éclairer les discussions en cours (hypothèses évoquées en introduction de différenciation modale ou aspectotemporelle pour expliquer la distribution des deux formes).

15. Pondérant la répartition FP/FS dans l'ensemble de corpus, nous avons fixé comme objectif d'annoter 60 occurrences par genre pour le FP, et 50 par genre pour le FP. Lorsque le nombre total d'occurrences est inférieur, dans un genre donné, à l'objectif quantitatif fixé, nous avons essayé, lorsque cela était possible, de compenser les occurrences manquantes par l'annotation d'occurrences d'un autre genre interactionnel mais appartenant au même sous-corpus ESLO1 ou ESLO2.

16. La décision est prise après analyse contextualisée de l'occurrence et évaluation de la compatibilité de l'autre forme avec le contexte. La prise en compte de la situation de communication, des interlocuteurs et leur visée communicative est ici décisive et nécessite 
souvent la consultation d'un contexte large qui passe la plupart du temps par l'écoute de longs extraits de corpus, qu'il n'est malheureusement pas possible de retranscrire ici dans leur intégralité. Les transcriptions alignées au son sont consultables sur le site d'ESLO.

17. Il s'agit, non pas d'une impossibilité de nature agrammaticale, mais d'une inadéquation avec le contexte.

18. Contexte interprétable comme celui du tour populaire "quand les poules auront des dents".

19. Wagner \& Pinchon (1991) rangent le FP parmi les périphrases exprimant la "phase antérieure au début de l'accomplissement du procès", à côté de être sur le point de et être à. Chez Riegel et al. (1994), aller est considéré comme l'un des auxiliaires aspectuels qui marquent le stade antérieur au début de la réalisation du procès, au même titre que être sur le point de, être en passe de. Wilmet (2007) parle de "sécant perspectif".

20. A huit heures, il va (être en train de) manger (Gosselin, 1996).

21. Des vérifications plus poussées semblent nécessaires puisque ce type d'emploi n'a pas, nous semble-t-il, été identifié en français (par exemple, Lansari (2009) n'en parle pas).

22. Fleischman (1983) parle de Present Relevance (la pertinence pour le présent), et observe que non seulement des formes rétrospectives par rapport au présent deviennent de nouveaux temps du passé, mais aussi que des formes prospectives finissent par constituer de nouveaux temps du futur. Wagner \& Pichon (1991 : 375) notent quant à eux : « à cette forme temporelle objective [i.e. le futur simple] qui isole pour ainsi dire l'avenir du présent, s'oppose en français la périphrase Aller + Infinitif (ou encore Je dois + Infinitif, il me faut + infinitif) qui représente l'avenir en genèse dans le présent ». Enfin Wilmet (2007 : 412) distingue le FP du FS en opposant un futur-pont (FP, en lien avec le moment de l'énonciation) et un futur-fossé (FS, détaché du moment de l'énonciation).

23. On pourrait naturellement penser qu'en même temps qu'il exprime un engagement, le procès de (27) est présenté comme certain, ce qui poserait la question de l'exclusion totale des valeurs d'annotation pour cet attribut. Il nous semble pourtant que contrairement aux trois premières valeurs, l'engagement présente une direction d'ajustement qui va de l'énoncé au monde, ce qui rend à notre avis peu petinente une classification en degrés de validation (certain, non-certain ...) censée mesurer la conformité de l'énoncé avec le monde. Si les énoncés (21) et (22) expriment une prédiction présentée comme certaine, la certitude dont il est question dans (27) ne peut pas être considérée comme un degré de validation d'une prédiction, mais un préalable à un engagement, qui ne peut pas être pris au sérieux autrement, censé faire correspondre le monde à l'énoncé.

24. Ces résultats entrent en contradiction avec ceux obtenus dans des études récentes sur des corpus de français parlé (cf. Bilger 2001, Jeanjean 1988), mais confirment les tendances dégagées par Emirkanian \& Sankoff (1986).

25. Ce regroupement permet d'isoler d'un côté les occurrences qui illustrent les valeurs modales des deux futurs (quelle que soit la part de futurité) et de l'autre les occurrences qui nous semblent dépourvues de ces valeurs modales. Cela permet pour la presente étude de se concentrer sur cette dernière catégorie, et d'interroger les hypothèses en cours sur les emplois futuraux.

26. Ces données ne semblent pas corroborer les hypothèses de Fleischman (1982) qui explique le renouvellement des formes du futur en diachronie par la progression des emplois modaux qui rendraient nécessaire la mobilisation d'une nouvelle forme pour l'expression de la futurité.

27. Compte tenu du nombre relativement restreint d'exemples annotés, nous sommes bien conscients que ces résultats n'ont pas de caractère définitif. D'autres tests sont à mener sur de plus larges cohortes d'exemples pour confirmer ces tendances et valider leur pertinence statistique. 


\section{RÉSUMÉS}

La présente étude traite du rapport synchronique et micro-diachronique entre les formes synthétique (FS) et analytique (FP) du futur en français moderne, sur la base d'un corpus oral échantillonné d'un million de mots, collecté à 40 ans d'intervalle (ESLO1 (1968-1971) et ESLO2 (2008-)). Les 3335 occurrences de futur identifiées ont fait l'objet d'une annotation manuelle multicouche qui visait à déterminer le type d'emploi (modal, futural et mixte), et à le sousspécifier pour un certain nombre de traits sémantiques (type de modalité, valeur aspectuelle, lien avec le présent...). Après une première étude consacrée au rapport FP/FS en lien avec la variable modale (Abouda \& Skrovec, 2015), nous nous proposons d'examiner ici la distribution synchronique et l'évolution micro-diachronique des emplois chronologiques (étiquetés « $\mathrm{f} »)$. L'annotation affinée des emplois permet de relier les tendances quantitatives microdiachroniques à des hypothèses qualitatives, l'occasion de discuter la pertinence des principales hypothèses avancées dans différents travaux pour expliquer le rapport FP/FS (différence aspectuelle, en termes de lien avec le présent, de proximité, validation).

This paper describes the synchronic and micro-diachronic relation between both synthetic (future simple) and analytic (future periphrastique) future tenses in modern French. This study is based on a sampled spoken corpus with 1 million words containing 2 datasets gathered in an interval of 40 years (ESLO1 1968-1971 and ESLO2 2008-). The 3335 found items were tagged according to their general semantic properties (expressing futurity, modality or both) as well as more specific properties (type of modality, aspect, present relevance...). Following a previous paper on modal features (Abouda \& Skrovec 2015), the present study aims at describing the temporal chronological use of both future tenses in order to highlight on one hand the synchronic distribution and on the other hand a possible diachronic change. We discuss some important hypothesis in the field (difference in aspect, regarding present relevance, chronologic proximity, validation).

\section{INDEX}

Mots-clés : futur simple, futur périphrastique, français, corpus oral, micro-diachronie, aspect, proximité avec le présent

\section{AUTEURS}

LOTFI ABOUDA

MARIE SKROVEC 